\title{
A Prospective Study Regarding the Need for Repeat Potassium Testing in ED Patients with Hemolysis- Associated Pseudo-Hyperkalemia
}

\author{
David Salo, Patrick Hinfey, Devansh Pandey, Renae Bernard, Randy Gill, Frederick Fiesseler
}

\section{ABSTRACT}

Objectives: Many ED patients who have hyperkalemia (HK) with evidence of hemolysis have pseudohyperkalemia (PHK) and may not require repeat potassium (K) testing, though ED physicians often redraw levels. We sought to determine if a set of patients with PHK could be determined who would not require repeat $\mathrm{K}$ draws based on lab and medication/co-morbid conditions.

Methods: This was a prospective IRB-approved study at an 85,000 patient/yr inner-city ED. Data was analyzed to determine whether repeat $\mathrm{K}$ draws were necessary in patients with HK plus hemolysis who had normal renal function (NFR) defined by either Cr or GFR and to determine factors that might lower retest threshold and prevent unnecessary repeat $K$ testing. Normal renal function was defined as $\mathrm{Cr} \leq \mathbf{1 . 3}$ or GFR $\geq 60$ (calculated by the Modification of Diet in Renal Disease (MDRD) equation: GFR $=186 \times \mathrm{SCr}^{-1.154} \times$ Age $^{-0.203}(\times 0.742$ if female $)$ or $(\times 1.21$ if black $)$. HK was defined as $\mathrm{K}>5.3 \mathrm{mEq} / \mathrm{L}$. ED patients with $\mathrm{K}$ draws showing HK plus hemolysis with repeat metabolic panel performed within 12 hours were eligible.

Results: 300 patients had HK with hemolysis with repeat testing during the study period. Four were excluded due to missing/incomplete data. Two patients had renal failure and were excluded. $238 \mathrm{had}$ repeat $K$ sent prior to, or without specific HK treatment and were further analyzed. The median age was 60, range 76, IQR 46-75. 153/238 (64.2\%) were female, 200/238 AfricanAmerican (84\%), 169/238 (71\%) had normal GFR while 182/238 (76.8\%) had normal Cr. Twenty-four of the $238(10 \%)$ had persistent HK on the $2^{\text {nd }}$ lab draw. Factors on univariate analysis associated with HK on repeat testing were age, gender, diuretic use, PVD, DM, and abnormal BUN, Cr or GFR. Binary logistic regression showed abnormal $\mathrm{Cr}$, age and $\mathrm{PVD}$ as significant predictors of persistent $\mathrm{HK}$. ROC curves of $\mathrm{Cr}$ and age were generated and further analysis showed $\mathrm{Cr}>1.4$, age $>63$, or PVD would have identified 22/24 cases of persistent hyperkalemia (the two patients showing persistent hyperkalemia had potassium levels that did not require treatment). This resulted in a decreased need for retesting in $53.7 \%$ of patients.

Conclusion: By using 3 variables: $\mathrm{Cr}>1.4$, age $>63$, and history of PVD, $115 / 214(53.7 \%)$ of patients with initial HK with hemolysis would not require repeat blood draw for potassium testing, while $2 / 24$ patients with persistent HK would have been missed. These 2 patients had marginally elevated potassium that would not have required treatment. Since some variables reported to be associated with hyperkalemia were not present or were present in low numbers, physicians may need to repeat testing on select or sicker patients who they suspect may have true HK, however up to $50 \%$ of patients will not require such testing.

Keywords: Hemolysis, pseudo-hyperkalemia, repeat lab work.
Submitted : December 16, 2021

Published : January 27, 2022

ISSN: $2593-8339$

DOI: $10.24018 /$ ejmed.2022.4.1.1175

\section{Salo*}

Morristown Medical Center, USA.

(e-mail: dave.salo@gmail.com)

P. Hinfey

Newark Beth Israel Hospital Newark, USA.

(e-mail: phinfey@protonmail.com) D. Pandey

Morristown Medical Center, USA. (e-mail:

dpandeydo2023@gmail.com)

R. Bernard

Newark Beth Israel Hospital, USA.

R. Gill

Newark Beth Israel Hospital, USA.

F. Fiesseler

Morristown Medical Center, USA.

(e-mail: ffiesseler@yahoo.com)

*Corresponding Author

\section{INTRODUCTION}

Emergency Department patients commonly have metabolic panels performed in the course of their evaluation and treatment. Hyperkalemia is a common electrolyte abnormality found in emergency department patients with potentially lethal side effects if not treated. Hyperkalemia is classified as mild (K 5.5-6.0 mEq/L), moderate (K 6.1-6.9 $\mathrm{mEq} / \mathrm{L})$ and severe $(\mathrm{K}>7.1 \mathrm{mEq} / \mathrm{L})[1]$. Pseudohyperkalemia, secondary to hemolysis, is occasionally found in patients with normal renal function and no history of renal disease or diseases affecting the kidneys. The metabolic panel is commonly repeated in patients with pseudohyperkalemia to be certain that true hyperkalemia is not present.

One retrospective study by Lombardi estimated that by using a creatinine $(\mathrm{Cr})$ based level for normal renal function (NRF) $23 \%$ of patients with $\mathrm{HK}$ may not require retesting whereas a second retrospective study, by Nguyen, suggested no patients with normal $\mathrm{Cr}$ require further testing [2], [3]. Gupta however stated that since a true positive rate of 5\% for severe hyperkalemia exists with normal $\mathrm{Cr}$ that all patients required further testing [4]. Since repeat blood testing causes 
increased patient discomfort, adds to length of stay (1.5 hours/redraw) and increased cost (\$20/test) it was felt cost savings could be realized if it was possible to reliably identify patients who do not need repeat testing [2]. We performed a prospective analysis of patients with hyperkalemia with hemolysis in an ED setting to determine if certain factors could predict patients at low or high risk for repeat hyperkalemia.

\section{A. Study Setting and Population}

The setting was the adult emergency department (ED) of an inner-city hospital with an emergency medicine residency program and a combined adult and pediatric annual volume of 85,000 patients (adult visits 55,000/year). Eighty-five to ninety-five percent of patients presenting to this ED are African-American.

\section{B. Study Protocol}

This was a prospective study on consecutive ED patients presenting with hyperkalemia from 9/27/2005-2/24/2006. The hospital's laboratory computer database was queried daily to identify ED patients who had metabolic panels performed that showed elevated potassium with hemolysis, and in whom the metabolic panel was repeated within 12 hours. The hospital's Institutional Review Board approved the study. Exclusion criteria: patients with incomplete data information; treatment of HK prior to repeat $\mathrm{K}$ level with sodium bicarbonate, glucose, insulin, albuterol or calcium (patients treated with oral or intravenous hydration were not excluded); active renal failure requiring dialysis. A study coordinator worked 5 days/week, $8 \mathrm{am}-5 \mathrm{pm}$ and collected data in real time during these shifts. In addition, each morning the lab provided a list of ED patients with HK and hemolysis since the last active shift. The study coordinator or ED residents involved in the study completed data collection for these patients. The principal investigator (PI) met with the study coordinator weekly to answer questions and ensure proper data handling. Patient charts were obtained from the ED's electronic medical record or the medical records department and patients were interviewed when available to complete data collection. Data was abstracted regarding past medical history, medications, and lab results, and recorded on a structured data abstraction form. Data abstractors were trained in use of the data forms to ensure accuracy and consistency. A copy of the data extraction form is listed in Appendix A.

\section{Data Analysis}

Data was entered into a Microsoft Excel database, (Version 14, 2010 Microsoft, Redmond, WA) and analyzed using STATGRAPHICS Centurion XVI (Version 16.1.11, StatPoint Technologies Incorporated, Warrenton, VA) Analyze-It Software (Analyze-IT Software Ltd, Version 2.24, Leeds, UK) and MedCalc (Version 12.0.4.0 Mariakerke, Belgium). Data was analyzed to determine whether repeat $\mathrm{K}$ draws were necessary in patients with $\mathrm{HK}$ plus hemolysis. This was defined as persistent $\mathrm{HK}$ on the $2^{\text {nd }}$ draw (degree or evidence of hemolysis was not recorded for the $2^{\text {nd }}$ draw). We sought to identify factors that might lower the retest threshold in patients with persistent HK on repeat testing when renal function was normal utilizing univariate analysis and binary logistic regression. These included GFR,
Cr, BUN, Age, DM, HTN, history of renal insufficiency, history of CHF, PVD, use of K sparing diuretics, use of ACE inhibitors, use of ARBs, use of $\mathrm{K}$ salts, use of any diuretics, and NSAID use. HK was defined as $\mathrm{K}>5.3 \mathrm{mEq} / \mathrm{L}$. Abnormal $\mathrm{Cr}$ was defined as $>1.3 \mathrm{mg} / \mathrm{dL}$ whereas abnormal BUN was $>21 \mathrm{mg} / \mathrm{dL}$. NRF was defined as $\mathrm{Cr}<1.3$ or GFR $\geq 60$ (calculated by the Modification of Diet in Renal Disease (MDRD) equation

$$
G F R=186 \times S C r^{-1.154} \times \mathrm{Age}^{-0.203}(\times
$$

0.742 if female $)$ or $(\times 1.21$ if black $)$. ED patients with $\mathrm{K}$ levels indicating HK plus hemolysis, with a repeat metabolic panel performed within 12 hours were eligible. Appropriate parametric or nonparametric tests for continuous variables were employed for univariate analysis. For categorical variables either the Chi-square or Fishers Exact test was used. A p value of 0.05 was considered significant. Receiver Operator Curves were generated to determine optimal cutoffs for modeling when continuous variables were utilized.

\section{RESUlts}

Three hundred patients had HK with hemolysis with repeat $\mathrm{K}$ testing during the study period. Four patients were excluded due to missing/incomplete data whereas two patients were excluded for renal failure. Two hundred and thirty eight patients had repeat $\mathrm{K}$ sent prior to, or without $\mathrm{HK}$ treatment and were further analyzed. Median age was 60 , range 76, IQR 46-75. 153/238 (64.2\%) were female, 200/238 African-American (84\%), 169/238 (71\%) had normal GFR whereas $182 / 238$ (76.8\%) had normal Cr. Twenty four of the $238(10 \%)$ had persistent HK on a repeat lab draw.

\begin{tabular}{ccc}
\multicolumn{2}{c}{ TABLE I: STUDY GROUP CHARACTERISTICS } \\
\hline \hline Variable & Enrolled & $(\mathrm{n})=238$ \\
\hline \hline Female & 153 & $(64.2 \%)$ \\
Normal Cr & 182 & $(76.8 \%$ \\
Normal GFR & 169 & $(71.9 \%)$ \\
HTN & 129 & $(54.2 \%)$ \\
DM & 79 & $(33.2 \%)$ \\
History CHF & 31 & $(13.0 \%)$ \\
K Sparing Diuretics & 5 & $(2.1 \%)$ \\
ACE inhibitors & 47 & $(19.7 \%)$ \\
ARB & 8 & $(3.4 \%)$ \\
Any diuretic use & 48 & $(20.2 \%)$ \\
Use of K salts & 19 & $(8.0 \%)$ \\
NSAID's & 48 & $(20.2 \%)$ \\
PVD & 10 & $(4.2 \%)$ \\
\hline \hline
\end{tabular}

Table I shows the number of patients with each variable of interest. Variables listed on the data form not included in the table were excluded secondary to no or very low numbers of patients with that condition.

Table II is the univariate analysis of variables for conditions associated with persistent HK on repeat blood draw and shows significant variables including age, abnormal GFR, abnormal Cr, abnormal BUN, DM, PVD and diuretic use. Since more patients had a normal $\mathrm{Cr}$ than a normal GFR, data was analyzed with $\mathrm{Cr}$ for cases of persistent HK.

Table III shows multivariate analysis of Cr, PVD and age as significant for persistent HK on repeat testing. All other variables on binary logistic regression were not significant. 
Table II: UNIVARIATE ANALYSIS OF FACTORS ASSOCIATED WITH REPEAT ABNORMAL K

\begin{tabular}{cccc}
\hline \hline History & Odds Ratio & $95 \%$ CI & p-value \\
\hline \hline Abnl* Cr & 8.9 & $3.57-22.32$ & 0.0001 \\
Abnl BUN & 9.6 & $3.16-29.1$ & 0.0001 \\
PVD & 6.9 & $1.80-26.6$ & 0.022 \\
Abnl GFR & 7.56 & $2.93-19.3$ & 0.0001 \\
Any diuretic & 4.05 & $1.68-9.74$ & 0.005 \\
DM & 3.91 & $1.63-9.39$ & 0.004 \\
Gender & 2.33 & $0.99-5.46$ & 0.08 \\
Use of K salts & 3.76 & $1.22-11.57$ & 0.06 \\
Renal & 4.56 & $1.29-16.13$ & 0.06 \\
insufficiency & 2.21 & $0.08-5.55$ & 0.13 \\
HTN & 3.15 & $0.06-16.56$ & 0.38 \\
Use of ARB & 2.28 & $0.25-21.29$ & 0.83 \\
K sparing & & & \\
diuretics & 1.40 & $0.53-3.77$ & 0.66 \\
Use ACE & 1.37 & $0.51-3.65$ & 0.69 \\
Inhibitor & 1.90 & $0.65-5.53$ & 0.37 \\
NSAID use & & & K Normal \\
History of CHF & Repeat K & Abnl Repeat & 0.0003 \\
QI & $73.1+/-$ & $58.5+/-18.6$ &
\end{tabular}

All based on 238 patients unless otherwise indicated

* Abnormal $=$ Abnl; QI $=$ Quantitative Information

TABLE III: MULTIVARIATE ANALYSIS OF CHARACTERISTICS ASSOCIATED WITH ABNORMAL REPEAT K LEVEL

\begin{tabular}{cc}
\hline \hline Characteristic & $p$-value \\
\hline \hline Cr Normal & 0.0001 \\
PVD & 0.03 \\
Age & 0.03 \\
\hline \hline
\end{tabular}

We created ROC curves based on abnormal repeat K levels plotted against age and $\mathrm{Cr}$. While we chose an abnormal $\mathrm{Cr}$ of 1.3 as a cutoff for our general analysis, we examined the ROC curve for $\mathrm{Cr}$ with persistent abnormal $\mathrm{K}$ to determine if the defined level differed from an optimal found on analysis.

ROC analysis for age showed a sensitivity of 75\% (53.3$90.295 \% \mathrm{CI}$ ) and specificity of 57\% (50.1-63.795\% CI) for persistent $\mathrm{HK}$ at age $>63$ years. ROC analysis also showed a $\mathrm{Cr}>1.4$ to have a sensitivity of $54 \%(2.8-74.4 \% 95 \% \mathrm{CI})$, and specificity of $85.9 \%(80.5-90.3 \% 95 \% \mathrm{CI})$ for persistent HK. We therefore used the combination $\mathrm{Cr}>1.4$, presence of PVD and age $>63$ to analyze the proportion of patients who would not require additional testing and the number of repeat positive hyperkalemia patients when patients with these factors were excluded. With these factors we found 22/24 patients with persistent hyperkalemia would have been identified whereas 115 of 214 patients with pseudohyperkalemia would not have required repeat testing; a decrease of $53.7 \%$. Of the 2 patients with persistent hyperkalemia on repeat testing one had a potassium of 5.5 $\mathrm{mEq} / \mathrm{L}$ (initial $\mathrm{K}^{+}$was $6.5 \mathrm{mEq} / \mathrm{L}$ ) and one $5.6 \mathrm{mEq} / \mathrm{L}$ (initial $\mathrm{K}^{+}$was $6.8 \mathrm{mEq} / \mathrm{L}$ ), both of which would likely not require treatment.

\section{DISCUSSION}

Hyperkalemia is a potentially life-threatening condition that often requires immediate intervention. Pseudohyperkalemia however can result from shearing or hemolysis of red blood cells during peripheral blood draw, thrombocytosis, or leukocytosis. Two independent and sequential mechanisms: the degranulation of platelets (which increases the potassium load to serum at the time of clot formation in vitro), and transfer of a part of this potassium load back into blood cells to maintain electrolyte and osmotic homeostasis have been explained as mechanisms responsible for pseudohyperkalemia [5].

Contributing to this are inappropriate collection techniques, use of unsuitable collection supplies, collection tube factors and specimen processing, handling and transport problems [6]. Several ED studies have retrospectively examined pseudohyperkalemia in the ED setting and came to different conclusions regarding the need for repeat potassium testing [4], [5], [6]. Lombardi reported factors associated with HK were: arrhythmias, numbness; chronic renal failure; current use of a loop diuretic, spironolactone, or an ACE inhibitor; or an abnormal BUN or Cr level. He concluded that patients without any of these risk factors are at extremely low risk for having $\mathrm{HK}$ and that $23 \%$ of patients had no risk factors and did not need a blood redraw. Lombardi suggested this phenomenon should be prospectively studied. Gupta recently reported that, without taking other factors into account, that a normal $\mathrm{Cr}$ in a hemolyzed specimen predicted normokalemia, but that a true positive rate of 5\% for severe hyperkalemia existed. Gupta suggested all patients should therefore have repeat testing until this problem could be further elucidated [4]. This is the first study to prospectively examine factors related to persistent $\mathrm{HK}$ in patients with an initial elevated potassium level.

Our study found that using $\mathrm{Cr}>1.4$, age $>63$ or a history of PVD identified 22/24 patients with persistent hyperkalemia on repeat blood testing and that of $2 / 24$ who were not identified, neither had repeat $\mathrm{K}$ requiring treatment or, if the physician chose to treat, admission [7]. The reduction in the need for repeat blood draws was $53.7 \%$. Of note, we did not examine whether similar characteristics could be applied to patients who have no evidence of hemolysis on initial blood draw who present with these factors. Since hemolysis might not be reported in all patients with pseudohyperkalemia, an even greater savings could be realized if these factors can be utilized for pseudohyperkalemia when no hemolysis is present. We used a strict definition of hyperkalemia at a level of $5.3 \mathrm{mEq} / \mathrm{L}$ though the need to treat true hyperkalemia may be much higher than the low retest threshold as defined by our study. Use of a higher threshold could result in an even further reduction in the need for repeat testing. Since hyperkalemia is a potentially life-threatening condition, some retrospective studies have suggested continued retesting of all patients. Our study suggests this is not necessary and that most patients can be identified using $\mathrm{Cr}>1.4$, age $>63$ or a history of PVD, in conjunction with the severity of patient illness. Since several factors noted to be associated with HK were in low numbers in our study, physicians should use their clinical judgment to determine when patients should be retested despite a lack of factors. Finally, this study should be validated with a prospective study.

\section{LiMitATIONS}

Our study was limited by several factors. By definition we decided to use the criterion of an abnormal repeat $\mathrm{K}$ level as an indication that the first level was not caused by pseudohyperkalemia. Since most physicians would be unwilling to do a $3^{\text {rd }}$ or $4^{\text {th }}$ blood draw, even if mild 
hyperkalemia with or without hemolysis were present on a $2^{\text {nd }}$ draw, this represents a working ED environment. This means however, that we may have overestimated the true overall and persistent $\mathrm{HK}$ rates, which could have affected age and $\mathrm{Cr}$ analysis.

Despite this, a substantial number of patients still will not require additional testing using our criteria. A fair number of patients were also treated for hyperkalemia after the first draw or received IV fluids, which may have altered or decreased the potassium level. Excluding these patients, or possibly lowering potassium levels with fluid treatment, may also have affected data analysis. In addition, there may have been patients with normal $\mathrm{Cr}$ levels with $\mathrm{HK}$ with hemolysis who the ED physician decided not to redraw a potassium level on. This however would likely have further decreased the proportion of patients who would not require repeat $\mathrm{K}$ levels identified by the 3 factors. Finally, this study was undertaken in an inner city hospital and $85 \%$ of patients were AfricanAmerican. Racial differences in relation to disease processes or medication side effects may have impacted the results of this study.

\section{CONCLUSIONS}

The majority of patients with persistent hyperkalemia requiring treatment found on repeat blood draw, after initial testing showed hyperkalemia plus hemolysis, can be identified using $\mathrm{Cr}>1.4$, age $>63$ or a history of PVD. Utilizing these criteria, patient illness and in conjunction with physician judgment, up to $53.7 \%$ of patients with pseudohyperkalemia will not require a repeat blood draw.

\section{DisClOSURE STATEMENT}

The authors have received no funding from any company or organization that would benefit from this article and have no relevant affiliations beyond their academic appointments.

\section{APPENDIX A}

TABLE IV: MEDICAL HISTORY LIST

\begin{tabular}{ccc}
\hline \hline Medical History & & \\
\hline \hline None & $\mathrm{Y}$ & $\mathrm{N}$ \\
Renal Failure & $\mathrm{Y}$ & $\mathrm{N}$ \\
Renal Insufficiency & $\mathrm{Y}$ & $\mathrm{N}$ \\
Polycystic Kidney Disease & $\mathrm{Y}$ & $\mathrm{N}$ \\
Solitary Kidney & $\mathrm{Y}$ & $\mathrm{N}$ \\
Adrenal Diseases & $\mathrm{Y}$ & $\mathrm{N}$ \\
Hypertension & $\mathrm{Y}$ & $\mathrm{N}$ \\
Diabetes Mellitus & $\mathrm{Y}$ & $\mathrm{N}$ \\
Congestive Heart Failure & $\mathrm{Y}$ & $\mathrm{N}$ \\
MI/CAD & $\mathrm{Y}$ & $\mathrm{N}$ \\
Stroke/TIA & $\mathrm{Y}$ & $\mathrm{N}$ \\
Peripheral Vascular Disease & $\mathrm{Y}$ & $\mathrm{N}$ \\
Asthma/COPD & $\mathrm{Y}$ & $\mathrm{N}$ \\
HIV/AIDS & $\mathrm{Y}$ & $\mathrm{N}$ \\
Renal Tubular Acidosis & $\mathrm{Y}$ & $\mathrm{N}$ \\
Other & $\mathrm{Y}$ & $\mathrm{N}$ \\
\hline \hline
\end{tabular}

TABLE V: MEDICATIONS LIST

\begin{tabular}{ccc}
\hline \hline Medications & & \\
\hline \hline ACE Inhibitors & $\mathrm{Y}$ & $\mathrm{N}$ \\
ARB's & $\mathrm{Y}$ & $\mathrm{N}$ \\
K+-Sparing Diuretics & $\mathrm{Y}$ & $\mathrm{N}$ \\
Digitalis & $\mathrm{Y}$ & $\mathrm{N}$ \\
Diuretics & $\mathrm{Y}$ & $\mathrm{N}$ \\
Potassium Salts & $\mathrm{Y}$ & $\mathrm{N}$ \\
B-Blockers & $\mathrm{Y}$ & $\mathrm{N}$ \\
NSAID's & $\mathrm{Y}$ & $\mathrm{N}$ \\
Cyclosporine & $\mathrm{Y}$ & $\mathrm{N}$ \\
Arginine & $\mathrm{Y}$ & $\mathrm{N}$ \\
Heparin & $\mathrm{Y}$ & \\
TABLE VI: ED TREATMENTS LIST & \\
\hline \hline Treatments Given in the ED & & \\
\hline \hline oral fluids & $\mathrm{Y}$ & $\mathrm{N}$ \\
IV fluids & $\mathrm{Y}$ & $\mathrm{N}$ \\
calcium & $\mathrm{Y}$ & $\mathrm{N}$ \\
insulin & $\mathrm{Y}$ & $\mathrm{N}$ \\
sodium bicarbonate & $\mathrm{Y}$ & $\mathrm{N}$ \\
glucose & $\mathrm{Y}$ & $\mathrm{N}$ \\
B-agonists & $\mathrm{Y}$ & $\mathrm{N}$ \\
kayexalate & $\mathrm{Y}$ & $\mathrm{N}$ \\
hemodialysis & $\mathrm{Y}$ & \\
none & $\mathrm{Y}$ & \\
\hline \hline
\end{tabular}

\section{REFERENCES}

[1] Ahee, P. Crowe AV. The management of hyperkalemia in the emergency department. J Accid Emerg. Med. 2000; 17(3): 188-91.

[2] Lombardi AK. Miller CD. Johnson J. Chen N. Bahner D. Defining a set of criteria to reduce blood redraws in emergency department patients with pseudohyperkalemia. Acad Emerg. Med. 2005; 12(5): 79, Suppl 1.

[3] Nguyen T, Caraballo H, Buccelletti F. Hyperkalemia from hemolyzed specimens: must they be repeated. Ann Emerg. Med. 20006; 48(4): S100.

[4] Gupta A. Nguyen T. Husk G. Hyperkalemia in emergency department hemolyzed samples : must they be repeated ? Acad Emerg. Med. 2011; 18(5), S206.

[5] Sevatos,N. Theodossiades G, Archimandritis, A. Pseudohyperkalemia in serum: A new insight into an old phenomenon. Clin Med \& Res. 2008; 6(1): 30-32.

[6] Stankovic, AK. Smith, S. Elevated serum potassium values: The role of preanalytic variables. Am J Clin Pathol. 2004; 121: S105-12.

[7] Charytan, D. Goldfarb, DS. Indications for hospitalization of patients with hyperkalemia. Arch Intern Med. 2000; 160(11): 1605-11. 